\title{
Piet de Boer, Steven J. Morgan, Christian van der Werken: AO handbook orthopedic trauma care
}

\author{
Thieme Verlag, New York, Stuttgart, 2009, 400 p, 620 figs, 190 mm, Euro (D) 84,95 \\ CHF 141,00, ISBN: 978-3-13-146871-0
}

\author{
Claude Karger
}

Received: 20 December 2010/Accepted: 21 December 2010/Published online: 30 December 2010

(C) Springer-Verlag 2010

This handbook continues the AO tradition of excellence in medical education. It offers a thorough overview, explaining in its first part the fundamentals of orthopedic trauma care such as wound and fracture healing, principles of fracture treatment, and management of polytrauma patients.

The second part of the manual is devoted to the most frequent anatomical locations of injuries of the upper limb, the lower limb, as well as spine and pelvis lesions.

The third part deals with complications, compartment syndrome, and ligament instabilities. A final chapter is devoted to clinical examination and patient's history documentation.
The book is nicely structured in a pocket format; it is clearly presented, well illustrated, and concise. It offers clear definitions, practical notes with background informations, and hands-on instructions for patient treatment.

It will be a very welcome addition to all residents as they become more experienced in the assessment and management of traumatic injuries to the musculoskeletal system. It is an excellent introduction to orthopedic trauma care and will be well appreciated by every resident and fellow, at any level of training.

Conflict of interest No funds were received in support of this study.

C. Karger ( $\square)$

Strasbourg, France

e-mail: karger.claude@wanadoo.fr 